\title{
Design and Characteristic Analysis of a New Type of Hydraulic Pulsation Suppressor
}

\author{
Jun Yuan ${ }^{1}$, Hang Jiang ${ }^{1}$, Zheng-yan Fang ${ }^{2}$, Tian-sheng Wang ${ }^{1}$, Zhi Luo ${ }^{1}$, Feng-xian Jiang ${ }^{3}$ and Gui-hong Yin ${ }^{1}$ \\ ${ }^{1}$ Institute of Physics and Information Engineering, Zhaotong University, Zhaotong, Yunnan 657000 \\ ${ }^{2}$ School of Education and Science, Zhaotong University, Zhaotong, Yunnan 657000 \\ ${ }^{3}$ Business School, Yuxi Normal University Yuxi, Yunnan 653100
}

\begin{abstract}
In order to decrease the pressure pulsation of high-pressure hydraulic system and speed up the process of high-pressure hydraulic system, a new type of hydraulic pulsation suppressor is designed. The hydraulic pulsation suppressor is designed on the basis of the structural characteristics and attenuation performance of the expansion chamber attenuator and H-type muffler. The theoretical model of the modern hydraulic pulsation suppressor is established based on the fluid theory, and the attenuation performance of the new hydraulic pulsation suppressor is analyzed. The results demonstrate that the designed new hydraulic pulsation suppressor can attenuate the pressure pulsation with a pulsation frequency of $20 \mathrm{~Hz} \sim 2000 \mathrm{~Hz}$, and attenuate the frequency bandwidth; the attenuation effect is above $50 \mathrm{~dB}$, and the attenuation effect is ideal.
\end{abstract}

\section{Introduction}

With the development of hydraulic technology, the pressure of the hydraulic system is further increased. High pressure has made the pressure pulsation problem of the hydraulic system more prominent, which has been one of the important factors hindering the development of hydraulic technology. Therefore, reducing the pressure pulsation of the high-pressure hydraulic system is of great importance.

At present, hydraulic pulsation attenuators are mainly used in hydraulic systems to attenuate the pressure pulsations $^{[1-5]}$. The hydraulic pulsation attenuator installed at the pump outlet mainly includes two types: resonance type and expansion chamber type. OU YANG Xiaoping ${ }^{[6]}$ summarized the resonant hydraulic pulsation attenuator, explained the research progress and performance of various types of resonant hydraulic pulsation attenuators, and proposed that the resonance is best for compactness, high versatility, and strong frequency adaptability. The development trend of the research focusses of hydraulic pulsation attenuator. Resonant hydraulic pulsation attenuators are mainly used to eliminate low-frequency and intermediate-frequency narrow-band pressure pulsations. Wang Yan ${ }^{[7]}$ and others focused on the types, research methods and progress of expansion chamber hydraulic pulsation attenuators, and on this basis, discussed the research trend of expansion chamber hydraulic pulsation attenuators, and proposed that expansion chamber hydraulic pulsation attenuators should be based on theoretical methods and structures. Improve and strengthen research. The attenuation characteristics of the expansion chamber hydraulic pulsation attenuator mainly depend on the expansion ratio and length. This type of hydraulic pulsation attenuator mainly attenuates the pressure pulsation in the high frequency band ${ }^{[8]}$.

Built on the above analysis, a new type of hydraulic pulsation suppressor combining expansion chamber type and resonance type is designed. The original hydraulic pulsation suppressor combines the advantages of the expansion chamber hydraulic pulsation attenuator and the H-type hydraulic silencer. In this study, a theoretical analysis of the latest hydraulic pulsation suppressor was carried out by means of fluid dynamics; the insertion loss was used to evaluate the attenuation performance of the new hydraulic pulsation suppressor. The rationality of the modern hydraulic pulsation suppressor is verified.

\section{The working principle of the new hydraulic pulsation suppressor}

Figure 1 is a schematic diagram of the structure of the new hydraulic pulsation suppressor. The original hydraulic pulsation suppressor is composed of a rigid shell, two expansion chambers, twelve volume chambers, twelve slender mass chambers and two flow-limiting pipes. When the pulsation of the plunger pump enters the first expansion chamber from the inlet of the suppressor, the pulsation is attenuated for the first time; after the first decay, the pulsation enters the second expansion chamber with different parameters through the first restrictor pipe, and then attenuates again; The second attenuated pulsation flows through the second restrictor pipe and then enters the first volume chamber through the four mass

\footnotetext{
* Corresponding author: 2451485983@qq.com
} 
chambers; the hydraulic oil entering the first volume chamber is divided into two parts, one part is compressed, and the other part enters the first volume chamber. Two volume chambers; the same is valid for the hydraulic oil entering the second volume chamber; at this time, the hydraulic oil entering the third volume chamber is only compressed. The hydraulic oil in the twelve mass chambers moves with the pulsation of the piston, plays the role of liquid sense and fluid resistance, and consumes a part of the pulsation energy at the same time. The expansion chamber, mass chamber, and volume chamber has different parameters, which can be used for multiple frequency ranges. The pressure pulsation is attenuated many times. Through the reasonable design of the modern hydraulic pulsation suppressor, a new type of hydraulic pulsation suppressor integrating expansion chamber, restrictor tube, mass chamber and volume chamber is realized.

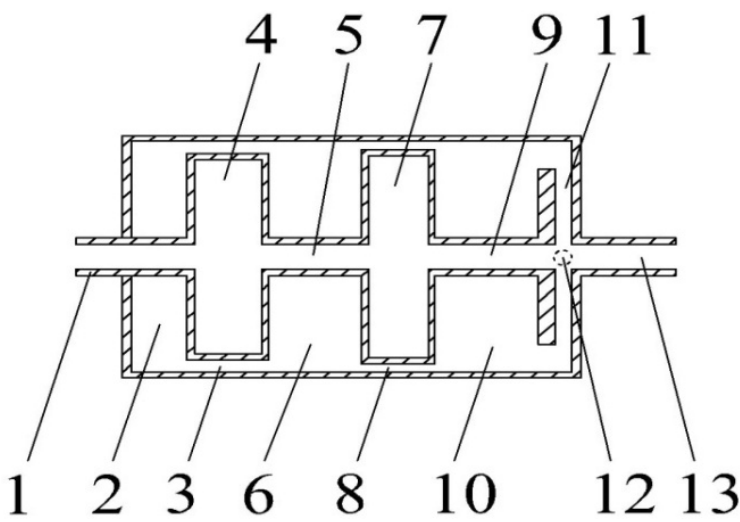

1. Suppressor inlet pipe 2. Volume chamber 3 3. Mass chamber 3 4. Expansion chamber 1 5. Flow restriction pipe $1 \quad 6$. Volume chamber 2 7. Expansion chamber 2 8. Mass chamber 2 9. Restriction pipeline 2 10. Volume chamber 1 11. Mass chamber 1

12. Node 1 13. Suppressor outlet pipeline

Figure 1. Schematic diagram of the structure of the new hydraulic pulsation suppressor.

\section{Mathematical model and attenuation characteristics analysis of the new hydraulic pulsation suppressor}

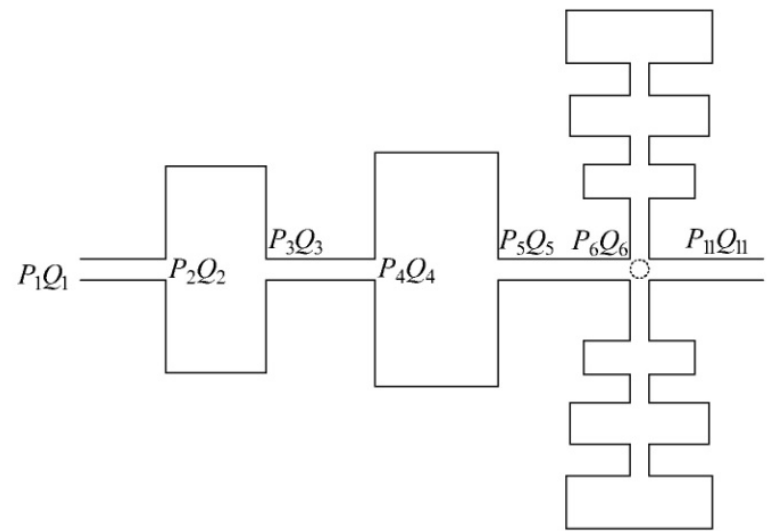

Figure 2. Simplified model diagram of the new hydraulic pulsation suppressor.
The simplified model of the new hydraulic pulsation suppressor is shown in Figure 2. In Figure 2, $P_{1}, Q_{1}$, and $P_{12}, Q_{12}$ are the frequency domain dynamic pressure and flow at the inlet and outlet of the new hydraulic pulsation suppressor; $P_{6}, Q_{6}$, and $P_{11}, Q_{11}$ are the frequency domain dynamic pressure and flow at the inlet and outlet of node $1 ; P_{2}, Q_{2}$, and $P_{3}, Q_{3}$ are the frequency domain dynamic pressure and flow at the inlet and outlet of the expansion chamber $1 ; P_{4}, Q_{4}$, and $P_{5}$, $Q_{5}$ are the frequency domain dynamic pressure and flow at the inlet and outlet of the expansion chamber 2 .

Figure 3 is an equivalent simplified model diagram of node 1. In Figure $3, Q_{x}$ and $P_{x}(x=7,8,9,10)$ are the frequency domain dynamic pressure and flow of node 1 .

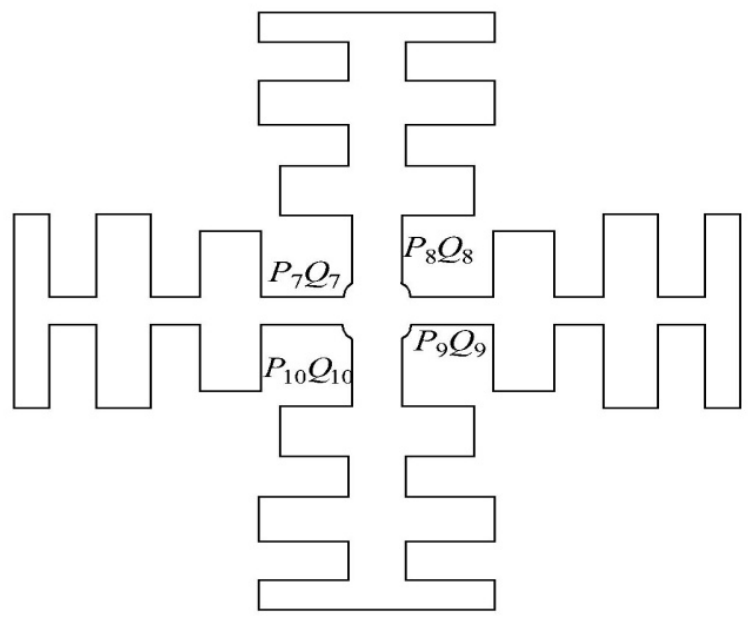

Figure 3. Simplified model diagram of node 1.

It can be written by node conditions:

$$
\begin{gathered}
Q_{6}=\sum_{\mathrm{x}=7}^{10} Q_{x}+Q_{11} \\
P_{6}=P_{7}=P_{8}=P_{9}=P_{10}=P_{11}
\end{gathered}
$$

Make

$$
Z_{x}=P_{x} / Q_{x}
$$

Where, $\quad x=7,8,9,10$

$Z_{x}$ is the branch point impedance of the three-stage H-type hydraulic silencer system. Figure 5 is a schematic diagram of the structure of a three-stage H-type hydraulic silencer. According to the literature [9], the expression of $Z_{x}$ is:

$$
Z_{x}=\frac{a_{11 x} a_{23 x} a_{32 x}+a_{12 x} a_{21 x} a_{33 x}-a_{11 x} a_{22 x} a_{33 x}}{\rho \operatorname{cs} A_{1 x}\left(a_{23 x} a_{32 x}-a_{22 x} a_{33 x}\right)}
$$




$$
\begin{gathered}
\text { Where, } a_{11 x}=\rho A_{1 x} l_{1 x} s^{2}+8 \pi \rho v l_{1 x} s+K \frac{A_{1 x}^{2}}{V_{1 x}} \\
a_{12 x}=a_{21 x}=-K \frac{A_{1 x} A_{2 x}}{V_{1 x}} \\
a_{23 x}=a_{32 x}=-K \frac{A_{2 x} A_{3 x}}{V_{2 x}} \\
a_{22 x}=\rho A_{2 x} l_{2 x} s^{2}+8 \pi \rho v l_{2 x} s+K A_{2 x}^{2}\left(\frac{1}{V_{1 x}}+\frac{1}{V_{2 x}}\right) \\
a_{33 x}=\rho A_{3 x} l_{3 x} s^{2}+8 \pi \rho v l_{3 x} s+K A_{3 x}^{2}\left(\frac{1}{V_{2 x}}+\frac{1}{V_{3 x}}\right)
\end{gathered}
$$$$
\left[\begin{array}{l}
P_{1} \\
Q_{2}
\end{array}\right]=\left[\begin{array}{ll}
A_{1} & B_{1} \\
C_{1} & D_{1}
\end{array}\right]\left[\begin{array}{cc}
1 & 0 \\
\frac{V_{2}}{K S} & 1
\end{array}\right]\left[\begin{array}{ll}
A_{3} & B_{3} \\
C_{3} & D_{3}
\end{array}\right]\left[\begin{array}{cc}
1 & 0 \\
\frac{V_{4}}{K S} & 1
\end{array}\right]\left[\begin{array}{cc}
A_{5} & B_{5} \\
C_{5} & D_{5}
\end{array}\right]\left[\begin{array}{c}
P_{6} \\
Q_{6}
\end{array}\right]
$$

Figure 4. $L$ is the length of the mass chamber; $A$ is the crosssectional area of the mass chamber; $V$ is the volume of the volume chamber;

The principle diagram of the three-stage H-type hydraulic pulsation attenuator.

The transfer matrix from the inlet pipe of the hydraulic pulsation suppressor to node 1 is:
Where, $\quad V_{2}$ is the volume of expansion chamber 1

$V_{4}$ is the volume of expansion chamber 2

For the pipeline, its dynamic characteristic equation is expressed as:

$$
\begin{array}{r}
{\left[\begin{array}{ll}
A_{i} & B_{i} \\
C_{i} & D_{i}
\end{array}\right]=\left[\begin{array}{cc}
\operatorname{ch}\left[T(s) L_{i}\right] & Z_{i}(s) \operatorname{sh}\left[T(s) L_{i}\right] \\
\operatorname{sh}\left[T(s) L_{i}\right] / Z_{i}(s) & \operatorname{ch}\left[T(s) L_{i}\right]
\end{array}\right]} \\
\text { Where, } \quad Z_{i}(s)=\rho c^{2} T(s) /\left(A_{x i} s\right) \text { is the }
\end{array}
$$
characteristic impedance of the pipeline

$$
\begin{aligned}
& T(s)=\left(s^{2}+R_{v} s\right)^{0.5} / c \text { is the pipe } \\
& \text { propagation constant } \\
& A_{x i} \text { is the pipe cross-sectional area } \\
& L_{i} \text { is the length of the pipe } \\
& R_{v}=32 \mu / A_{x i} \\
& i=1,3,5
\end{aligned}
$$

From the formula (5):

$$
\left[\begin{array}{l}
P_{1} \\
Q_{1}
\end{array}\right]=\left[\begin{array}{ll}
A_{11} & B_{11} \\
C_{11} & D_{11}
\end{array}\right]\left[\begin{array}{l}
P_{6} \\
Q_{6}
\end{array}\right]
$$

Simplify (7) formula, there are

$$
\begin{gathered}
P_{1}=A_{11} P_{6}+B_{11} Q_{6}=A_{11} P_{11}+B_{11} Q_{6} \\
Q_{1}=C_{11} P_{6}+D_{11} Q_{6}=C_{11} P_{11}+D_{11} Q_{6}
\end{gathered}
$$

From (1), (2) and (3), we get:

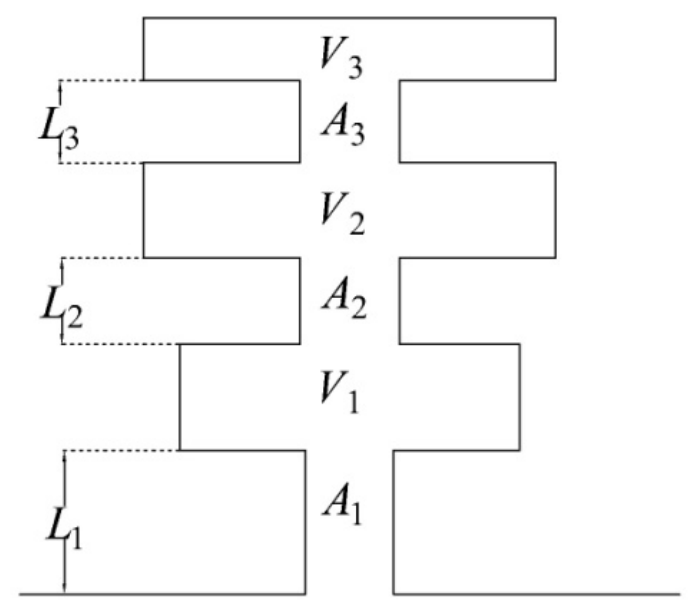

$$
Q_{6}=\sum_{\mathrm{x}=7}^{10} \frac{P_{x}}{Z_{x}}+Q_{11}=P_{11} \sum_{\mathrm{x}=7}^{10} \frac{1}{Z_{x}}+Q_{11}
$$

From (9) and (10), we get:

$$
Q_{1}=C_{11} P_{11}+D_{11}\left(P_{11} \sum_{\mathrm{x}=7}^{10} \frac{1}{Z_{x}}+Q_{11}\right)
$$

Make $Y=Q_{11} / P_{11}$.

Substituting into (11) formula:

$$
P_{11}=\frac{Q_{1}}{C_{11}+D_{11} \sum_{\mathrm{x}=7}^{10} \frac{1}{Z_{x}}+D_{11} Y}
$$

Incorporate the outlet pipe of the hydraulic pulsation suppressor into the dynamic impedance part. The dynamic characteristics of the pipeline and throttling load, the expression of load admittance $Y$ can be obtained as ${ }^{[10]}$ :

$$
Y=\frac{2 \Delta P_{F} C_{22}+Q_{F} D_{22}}{2 \Delta P_{F} A_{22}+Q_{F} B_{22}}
$$

Where, $\quad \Delta P_{F}$ is the pressure loss of the throttle valve

$Q_{F}$ is the flow rate of the throttle valve

$A_{22} 、 B_{22} 、 C_{22} 、 D_{22}$ are the load-end pipelines to transfer matrix elements 
The anti-vibration effect of the new hydraulic pulsation suppressor is evaluated by the insertion loss $K_{\mathrm{i}}$. The insertion loss not only considers the characteristics of the pulsation attenuator device and the piping system, but also considers the influence of source impedance and load impedance. The insertion loss is defined as the pressure ratio of the pipe load end before and after adding the pulsation attenuator ${ }^{[11]}$, namely

$$
K_{\mathrm{c}}=20 \lg \left|\frac{P_{11}^{\prime}}{P_{11}}\right|
$$

$P_{11}^{\prime}$ is the use of rigid hydraulic tubing of the same length as the pulsation attenuator to replace the frequency domain pressure at the load end of the new hydraulic pulsation suppressor.

$P_{1}^{\prime}, Q_{1}^{\prime}$ and $P_{11}^{\prime}, Q_{11}^{\prime}$ are the dynamic frequency domain pressure and dynamic frequency domain flow rate at the inlet and outlet of the rigid straight hydraulic oil pipe after replacing the hydraulic pulsation attenuator with a rigid straight hydraulic oil pipe. The dynamic characteristics of the rigid hydraulic oil pipe are obtained according to the pipeline network calculation method. The equation is:

$$
\left[\begin{array}{l}
P_{1}^{\prime} \\
Q_{1}^{\prime}
\end{array}\right]=\left[\begin{array}{ll}
A_{33} & B_{33} \\
C_{33} & D_{33}
\end{array}\right]\left[\begin{array}{l}
P_{11}^{\prime} \\
Q_{11}^{\prime}
\end{array}\right]
$$

From the formula (15):

$$
P_{15}^{\prime}=\frac{Q_{1}^{\prime}}{\left(C_{33}+D_{33} Y\right)}
$$

For the plunger pump with flow disturbance source, it's working condition is stable, so

$$
Q_{1}=Q_{1}^{\prime}
$$

Substituting (10), (16), and (17) into (14), the insertion loss of the new hydraulic pulsation suppressor can be expressed as:

$$
K_{\mathrm{c}}=20 \lg \left|\frac{C_{11}+D_{11} \sum_{\mathrm{x}=7}^{10} \frac{1}{Z_{x}}+D_{11} Y}{C_{33}+D_{33} Y}\right|
$$

\section{Simulation analysis of the attenuation characteristics of the new hydraulic pulsation suppressor}

Use MATLAB software to simulate the insertion loss of the original hydraulic pulsation suppressor. The basic parameters of the simulation are presented in table 1 . The parameters of the throttle valve and hydraulic oil are given in table 2. The simulation frequency range is: $0 \mathrm{~Hz} \sim 2000$

\begin{tabular}{|c|c|c|}
\hline & radius $r_{1} / \mathrm{mm}$ & 12.5 \\
\hline \multirow{2}{*}{ Quality Room 7} & Length $L_{7} / \mathrm{mm}$ & 60 \\
\hline & radius $r_{7} / \mathrm{mm}$ & 7 \\
\hline \multirow{2}{*}{ Quality Room 8} & Length $L_{8} / \mathrm{mm}$ & 60 \\
\hline & radius $r_{8} / \mathrm{mm}$ & 6 \\
\hline \multirow{2}{*}{ Quality Room 9} & Length $L_{9} / \mathrm{mm}$ & 60 \\
\hline & radius $r_{9} / \mathrm{mm}$ & 5 \\
\hline \multirow{2}{*}{ Quality Room 10} & Length $L_{10} / \mathrm{mm}$ & 60 \\
\hline & radius $r_{10} / \mathrm{mm}$ & 4 \\
\hline \multirow{2}{*}{ Restrictor pipe 1} & Length $L_{\mathrm{x} 1} / \mathrm{mm}$ & 23 \\
\hline & radius $r_{\mathrm{x} 1} / \mathrm{mm}$ & 12.5 \\
\hline \multirow{2}{*}{ Restrictor pipe 3} & Length $L_{\mathrm{x} 3} / \mathrm{mm}$ & 23 \\
\hline & radius $r_{\mathrm{x} 3} / \mathrm{mm}$ & 12.5 \\
\hline \multirow{2}{*}{ Restrictor pipe 5} & Length $L_{\mathrm{x} 5} / \mathrm{mm}$ & 23 \\
\hline & radius $r_{\mathrm{x} 5} / \mathrm{mm}$ & 12.5 \\
\hline \multirow{2}{*}{ Expansion room 1} & Length $L_{\mathrm{k} 1} / \mathrm{mm}$ & 120 \\
\hline & radius $r_{\mathrm{k} 1} / \mathrm{mm}$ & 90 \\
\hline \multirow{2}{*}{ Expansion room 2} & Length $L_{\mathrm{k} 2} / \mathrm{mm}$ & 100 \\
\hline & radius $r_{\mathrm{k} 2} / \mathrm{mm}$ & 90 \\
\hline \multirow{3}{*}{ Volume chamber 1} & Length $L_{\mathrm{v} 1} / \mathrm{mm}$ & 23 \\
\hline & Inner radius $r_{\mathrm{n} 1} / \mathrm{mm}$ & 12.5 \\
\hline & Outer radius $r_{\mathrm{wl}} / \mathrm{mm}$ & 100 \\
\hline \multirow{3}{*}{ Volume chamber 2} & Length $L_{\mathrm{v} 2} / \mathrm{mm}$ & 23 \\
\hline & Inner radius $r_{\mathrm{n} 2} / \mathrm{mm}$ & 12.5 \\
\hline & Outer radius $r_{\mathrm{w} 2} / \mathrm{mm}$ & 100 \\
\hline \multirow{3}{*}{ Volume chamber 3} & Length $L_{\mathrm{v} 3} / \mathrm{mm}$ & 23 \\
\hline & Inner radius $r_{\mathrm{n} 3} / \mathrm{mm}$ & 12.5 \\
\hline & Outer radius $r_{\mathrm{w} 3} / \mathrm{mm}$ & 100 \\
\hline \multirow{2}{*}{ Quality Room 72} & Length $L_{72} / \mathrm{mm}$ & 100 \\
\hline & radius $r_{72} / \mathrm{mm}$ & 7 \\
\hline \multirow{2}{*}{ Quality Room 82} & Length $L_{82} / \mathrm{mm}$ & 100 \\
\hline & radius $r_{82} / \mathrm{mm}$ & 6 \\
\hline \multirow{2}{*}{ Quality Room 92} & Length $L_{92} / \mathrm{mm}$ & 100 \\
\hline & radius $r_{92} / \mathrm{mm}$ & 5 \\
\hline \multirow{2}{*}{ Quality Room 102} & Length $L_{102} / \mathrm{mm}$ & 100 \\
\hline & radius $r_{102} / \mathrm{mm}$ & 4 \\
\hline \multirow{2}{*}{ Quality Room 73} & Length $L_{73} / \mathrm{mm}$ & 120 \\
\hline & radius $r_{73} / \mathrm{mm}$ & 7 \\
\hline \multirow{2}{*}{ Quality Room 83} & Length $L_{83} / \mathrm{mm}$ & 120 \\
\hline & radius $r_{83} / \mathrm{mm}$ & 6 \\
\hline \multirow{2}{*}{ Quality Room 93} & Length $L_{93} / \mathrm{mm}$ & 120 \\
\hline & radius $r_{93} / \mathrm{mm}$ & 5 \\
\hline \multirow{2}{*}{ Quality Room 103} & Length $L_{103} / \mathrm{mm}$ & 120 \\
\hline & radius $r_{103} / \mathrm{mm}$ & 4 \\
\hline \multirow{2}{*}{$\begin{array}{l}\text { Load equivalent } \\
\text { pipeline }\end{array}$} & Length $L_{\mathrm{f}} / \mathrm{mm}$ & 400 \\
\hline & radius $r_{\mathrm{f}} / \mathrm{mm}$ & 12.5 \\
\hline
\end{tabular}
$\mathrm{Hz}$.

Table1. Basic parameter table of the new hydraulic pulsation suppressor.

\begin{tabular}{|c|c|c|}
\hline Name & Physical parameter & Value \\
\hline Inlet pipe & Length $L_{1} / \mathrm{mm}$ & 100 \\
\hline
\end{tabular}


Table2. Basic parameter table of hydraulic oil and throttle valve.

\begin{tabular}{|c|c|c|}
\hline Name & Physical parameter & Value \\
\hline \multirow{3}{*}{ No. 32 hydraulic oil } & Bulk modulus $/ \mathrm{MPa}$ & 1400 \\
\cline { 2 - 3 } & Viscosity $/(\mathrm{Pa} \cdot \mathrm{s})$ & 0.00314 \\
\cline { 2 - 3 } & density $/\left(\mathrm{kg} / \mathrm{m}^{3}\right)$ & 890 \\
\hline Throttle valve & $\begin{array}{c}\text { Differential pressure } \\
/ \mathrm{MPa}\end{array}$ & 2.5 \\
\cline { 2 - 3 } & $\mathrm{Flow} /(\mathrm{L} / \mathrm{min})$ & 160 \\
\hline
\end{tabular}

According to the above formula and the basic parameters of the simulation, the insertion loss of the hydraulic pulsation suppressor is programmed in MATLAB software as showed in Figure 5.

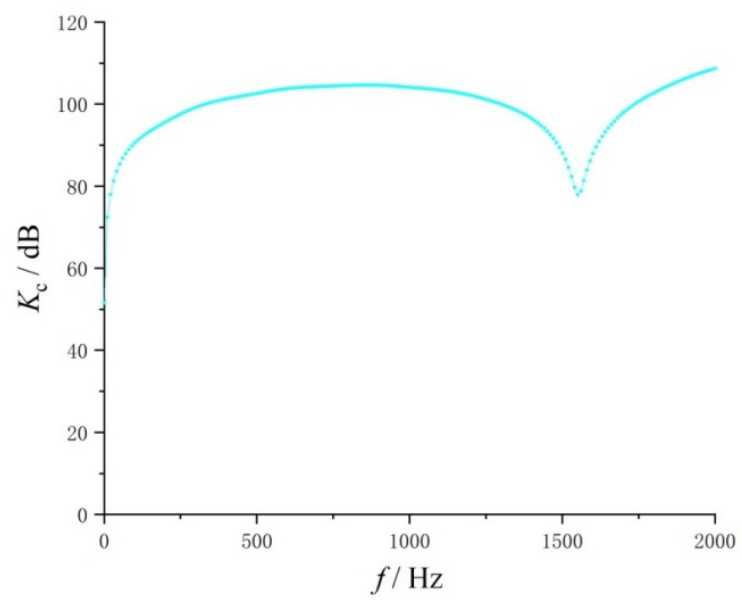

Figure 5. MATLAB simulation curve of insertion loss of the new hydraulic pulsation suppressor.

Figure 5 is the MATLAB simulation curve of the insertion loss of the original hydraulic pulsation suppressor. It can be obtained from the figure that the attenuation effect of the hydraulic pulsation suppressor is above $50 \mathrm{~dB}$ in the pulsation frequency range of $20 \mathrm{~Hz}$ to $2000 \mathrm{~Hz}$, and has a sound attenuation effect. Compared with ordinary pulsation attenuators, the attenuation effect is better and the attenuation frequency band is wider.

\section{Conclusion}

(1) A new type of hydraulic pulsation suppressor with compact structure is designed, and the mathematical model of the hydraulic pulsation suppressor is reasonably deduced. According to theoretical analysis, the new hydraulic pulsation suppressor achieves the effect of triple attenuation of pulsation in a wide frequency band.

(2) Through the analysis of the simulation calculation results of MATLAB software, it is found that the new hydraulic pulsation suppressor has an ideal effect of attenuating pressure pulsation in the frequency range of 20 $\mathrm{Hz}$ to $2000 \mathrm{~Hz}$, and achieves an efficient and broadspectrum attenuation of pressure pulsation.

\section{References}

1. HARRISON K A, EDGE K A.Reduction of axial piston pump pressure ripple[J]. Proceedings of the
Institution of Mechanical Engineers, Part I: Journal of Systems and Control Engineering, 2000, 214(1): 5364.

2. XU Bing, CHEN Yuan, ZHANG Jun-hui. Current Researches and Progress on Vibration and Noise Reduction Technology of Axial Piston Pump[J]. Chinese Hydraulics \& Pneumatics, 2014, (3): 1-12.

3. ZENG Xiangrong. Hydraulic noise control[M]. Harbin: Harbin Institute of Technology Press, 1988.

4. KWONG AH M, EDGE K A.A method to reduce noise in hydraulic systems by optimizing pipe clamp locations $[\mathrm{J}]$. Proceedings of the Institution of Mechanical Enginessrs, rat I: Journal of Systems and Control Engineering, 1998, 12(4): 67-280.

5. WANG Zhanlin. Aircraft high-pressure hydraulic energy system[M]. Beijing University of Aeronautics and Astronautics Press, 2004.

6. OUYANG Xiaoping, LI Lei, FANG Xu, YANG Huayong. Research Status and Prospects of Resonanttype Hydraulic Pulsation Attenuators[J]. Journal of Mechanical Engineering, 2015, 51(22): 168-175, 182.

7. WANG Yan, HAO Fengqian, GUO Shengrong, LU Yueliang, CHEN Jinhua. Research Status and Development Trend of the Expansion Chamber Pressure Pulsation Attenuator[J]. Machine Tool \& Hydraulics 2015, 43(15): 180-186.

8. YANG Fan, DENG Bin, WANG Guozhi, ea lt. Fluid Acoustic Properties of Expansion Chamber Mufflers Based on Electro-hydraulic Analogy Method[J]. Machine Tool \& Hydraulics, 2018, (14): 60-63.

9. GUAN Changbin, JIAO Zongxia. Modeling and Optimal Design of 3 Degrees of Freedom Helmholtz Resonator in Hydraulic System[J]. Chinese Journal of Aeronautics, 2012, 25(5): 776-783.

10. ZHANG Yin, YU Jun, LI Shen. Experimental research and CFD simulation of pressure pulsation attenuator[J].

Chinese Hydraulics \& Pneumatics, 2011, (6): 47-50.

11. SHAN Changji. Structure Design and Attenuation Method of Hydraulic Mufflers[J]. Journal of Jiamusi University(Natural Science Edition), 2013, (5): 749751. 